\title{
Immune Checkpoint Blockade for the Treatment of Hodgkin Lymphoma
}

\author{
Adam Yuh Lin (D)', Joseph Michael Schnitter², Leo I Gordon' \\ IDivision of Hematology Oncology, Department of Medicine, Feinberg School of Medicine, Northwestern University, Chicago, IL, USA; ${ }^{2}$ Department \\ of Medicine, Feinberg School of Medicine, Northwestern University, Chicago, IL, USA \\ Correspondence: Adam Yuh Lin, Division of Hematology Oncology, Department of Medicine, Feinberg School of Medicine, Northwestern University, \\ Arkes Pavilion, 676 N St Clair Street Suite 850, Chicago, IL, 606II, USA, Email adam.lin@northwestern.edu
}

\begin{abstract}
Classical Hodgkin lymphoma is biologically different than other lymphomas. The cancer cells only occupy a small amount of the lymph node and evade the immune system by amplification of PD-L1 and PD-L2. Therefore, checkpoint inhibitors are a logical treatment option for Hodgkin lymphoma patients to unlock the immune system. Checkpoint inhibitors have shown high response rates in clinical trials in advanced-stage Hodgkin lymphoma. The two most commonly used checkpoint inhibitors are pembrolizumab and nivolumab, both FDA approved as third-line therapy. There is increasing interest in the use of checkpoint inhibitors with combination chemotherapy or with other targeted agents in the second-line or even frontline setting. In this review, we will highlight the clinical trials that led to approvals of checkpoint inhibitors for Hodgkin lymphoma.
\end{abstract}

Keywords: checkpoint inhibitor, pembrolizumab, nivolumab, Hodgkin lymphoma

\section{Introduction}

Classical Hodgkin lymphoma (cHL) is largely curable with conventional chemotherapy, with an estimated 5-year survival of approximately $88 \% .{ }^{1}$ Treatment algorithms in $\mathrm{cHL}$ depend in part on the clinical stage at the time of diagnosis. In early-stage disease (stage I/II), using the National Comprehensive Cancer Network guidelines, patients are separated into favorable or unfavorable characteristics, depending on several factors including erythrocyte sedimentation rate, bulky disease (greater than one-third of the thoracic width or $\geq 10 \mathrm{~cm}$ in diameter) and number of nodal sites. ${ }^{2}$ Most early-stage patients can be cured by reduced cycles (two to four cycles) of chemotherapy combined with involved field radiation, though the optimal number of cycles and the need for combined modality therapy remain controversial. The chemotherapy regimen used in trials for early-stage disease is mostly ABVD (doxorubicin, bleomycin, vinblastine, and dacarbazine) with possible escalation to BEACOPP (bleomycin, etoposide, doxorubicin, cyclophosphamide, vincristine, procarbazine, and prednisone) if the interim positron emission tomography noted residual or progressive disease. The research focus for the treatment of early-stage disease $\mathrm{cHL}$ is to minimize therapy to mitigate the secondary cancer and cardiovascular risks. ${ }^{3,4}$

For advanced disease, frontline therapy for cHL has changed over the years. In addition to ABVD, BV-AVD (brentuximab vedotin, doxorubicin, vinblastine, and dacarbazine) has become an option, based on the results of the ECHLON-1 trial. ${ }^{5,6}$ Brentuximab vedotin (BV), which is an antibody drug conjugate, replaced bleomycin to reduce the risk of significant lung injury. A more intensive chemotherapy regimen, escalated BEACOPP, is often used in Germany, with similar results to ABVD. Despite this high response rate, a significant number of patients will not respond to frontline therapy or will have relapsed disease. Options for relapsed/refractory cHL patients include high-dose salvage intensive chemotherapy followed by high-dose chemotherapy and autologous hematopoietic stem cell transplantation (HSCT). The optimum second-line salvage chemotherapy regimen is not standardized and is still the subject of debate and investigation. Castagna et al detailed and categorized the second-line chemotherapy options into 1) platinum-based regimens such as ifosfamide, carboplatin, etoposide (ICE), ${ }^{7}$ with an overall response rate (ORR) of $72 \%$ and a complete 
response (CR) rate of $26 \%$; 2) gemcitabine-based regimens such as gemcitabine, vinorelbine, pegylated liposomal doxorubicin (GVD), ${ }^{8}$ with an ORR of $70 \%$ and a CR of $19 \%$; and 3) others, namely non-platinum or gemcitabine regimens. ${ }^{9}$ Our institution often uses ICE as salvage chemotherapy as it has the benefit of stem cell collection between cycles.

The unique biology of cHL can drive novel treatments. The malignant cell, the Reed-Sternberg cell (RSC), makes up only a small portion of the involved lymph nodes, while the majority are reactive T cells and other immune cells. Further evaluation of the RSCs found overexpression of PD-L1 and PD-L2 due to genetic amplification of the 9p24.1 locus. ${ }^{10}$

The overexpression of the PD-1 ligand leads to a T-cell-exhausted tumor microenvironment. ${ }^{11}$ The RSCs with copy number alterations of 9p24.1/CD274(PD-L1)/PDCD1LG2(PD-L2) are surrounded by PD-L1-positive tumor-associated macrophages. ${ }^{12}$ This is often described as a "castle and moat" model as the RSCs are surrounded by inactivated innate immune cells to protect them from the immune system, specifically, activated T cells. Importantly, alterations of PD-L1 and PD-L2 have correlated with reduced progression-free survival (PFS) when treated with standard chemotherapy regimens. ${ }^{13}$ Also, these RSCs had overall high mutational burden and microsatellite instability (MSI)-associated hypermutation, biological markers that correlate with the response to checkpoint inhibitor (CPI) therapy in other malignancies. ${ }^{14}$ Therefore, anti-PD-1/PD-L1 CPI therapy is a rational approach to the treatment of cHL. ${ }^{15-19}$ The success of PD-1 blockade suggests that some of these T cells are reversibly exhausted. RSCs with $\beta_{2}$-microglobulin/major histocompatibility complex (MHC) class I loss are noted to have more durable responses. ${ }^{11}$ In addition to MHC class I, newly diagnosed cHLs had a higher prevalence of NF-kB genetic alterations. Wienand et al also found a mutational signature of spontaneous deamination of cytosine-phosphate-guanines, apolipoprotein B mRNA editing catalytic polypeptide-like, activation-induced cytidine deaminase, and MSI-associated hypermutation. ${ }^{14}$

Clinical trial data have shown that anti-PD-1/PD-L1 monotherapy is an effective option for patients who have failed first-line chemotherapy. Thus far, two main CPIs - nivolumab and pembrolizumab, both of which are anti-PD-1 monoclonal antibodies - have been approved by the FDA for relapsed/refractory cHL. In this review, we will discuss the clinical trials for CPI in cHL, organized by the sequence of treatments.

\section{Third or Greater Line of Therapy}

In a 2015 study in which 23 patients with previously heavily treated (more than two lines of therapy) relapsed/refractory cHL were administered nivolumab every 2 weeks until they had evidence of CR, tumor progression, or severe toxicity, $80 \%$ of patients demonstrated an objective response, including $17 \%$ with CR and $70 \%$ with partial response (PR), with a rate of PFS of $86 \%$ at 24 weeks. ${ }^{20}$ A multicenter phase II study of nivolumab monotherapy in 17 Japanese patients with relapsed/refractory $\mathrm{cHL}$ who had all previously received BV demonstrated an objective response rate of $81.3 \%$ among the 16 patients included in the efficacy analysis. Among these 16 patients, four had complete remission and nine had partial remission. ${ }^{21}$ In another phase II study from 34 hospitals across Europe and North America, in which nivolumab monotherapy was administered to 80 patients with recurrent $\mathrm{cHL}$ who had progressed after autologous stem cell transplantation (ASCT) and either failed to respond to or relapsed following BV administration, 53 patients $(66.3 \%$, 95\% CI 54.8-76.4\%) demonstrated an objective response based on review from an independent radiological review committee (IRRC). ${ }^{22}$

The phase II CheckMate 205 trial also examined the efficacy of nivolumab in relapsed/refractory cHL, but further separated patients into three groups based on treatment history: BV-naïve, autologous hematopoietic cell transplantation (auto-HCT) followed by BV, and BV received before and/or after auto-HCT. ${ }^{23}$ In total, 243 patients were treated with nivolumab and the overall objective response rate was $69 \%$ (95\% CI 63-75\%), ranging from $65 \%$ to $73 \%$ in each cohort. The median response duration among all 243 patients was 16.6 months (95\% CI 12.2-20.3 months) and median PFS was 14.7 months (95\% CI 11.3-18.5 months). This trial, along with the 2015 trial, ${ }^{20}$ led to the FDA approval of nivolumab single agent for the treatment of relapsed cHL which had progressed after autologous HSCT and post-transplantation BV. Notably, this was the first FDA application for a PD-1 inhibitor in hematologic malignancies.

Around the same time, in 2017, the FDA also approved pembrolizumab for adults and children with cHL refractory to or which has relapsed after at least three prior therapies based on the initial results from KEYNOTE-087. ${ }^{24}$ The patients were divided into three cohorts: 1) patients with progression after ASCT and subsequent BV administration (n=69); 2) 
patients with progression following salvage chemotherapy and BV who were not candidates for ASCT ( $\mathrm{n}=81$ ); and 3) patients with progression after ASCT without exposure to BV $(\mathrm{n}=60)$. Cohort 1 had an ORR of $73.9 \%$ and a CR rate of $21.7 \%$. Cohort 2 had an ORR of $64.2 \%$ and a CR rate of $24.7 \%$. Cohort 3 had an ORR of $70 \%$ and a CR rate of $20 \%$. For all the patients included, the ORR was $69 \%$ and the CR rate was $22.4 \%$. A 5-year follow-up of KEYNOTE-087 reaffirmed the benefit of pembrolizumab in both BV-naïve patients and those with previous exposure. ${ }^{25}$ Out of the 210 patients evaluated, 46 patients completed 2 years of pembrolizumab. The ORR was $71 \%$, with a CR rate of $27.6 \%$. The ORR increased to $84.1 \%$ for cohort 1 and $67.9 \%$ for cohort 2, and there was a slight decrease to $68.3 \%$ for cohort 3 at the data cut-off. Furthermore, the median PFS was 56.5 months and the 5-year PFS rate was $44.3 \%$, while the median overall survival (OS) was not reached and the 5-year OS was impressive, at $82.8 \%$.

Before that trial, the phase Ib study KEYNOTE-013 helped open the door for the use of pembrolizumab in this population. ${ }^{23,26}$ The study consisted of 31 patients with relapsed/refractory cHL, all of whom had disease progression while on or after treatment with $\mathrm{BV}$, where $55 \%$ had more than four previous lines of therapy, and $71 \%$ had relapsed following ASCT. Among this group, the ORR was 65\% (90\% CI 48-79\%), with complete remission in $16 \%$ (90\% CI $7-31 \%$ ) and partial remission in $48 \%$ of patients. PFS was $69 \%$ at 24 weeks and $46 \%$ at 52 weeks.

Data from clinical trials have shown that anti-PD-1 therapies in combination with other agents are effective in relapsed/refractory cHL. A phase I trial investigated pembrolizumab plus vorinostat in patients with DLBCL, follicular lymphoma, and cHL who had failed one or more prior lines of treatment (with a median of four) and were not candidates for stem cell transplantation. ${ }^{27}$ In total, 30 patients were enrolled in the trial, 12 of whom had cHL. Among these 12, 11 had previously received BV, seven had been treated previously with anti-PD-1 therapy, and three were refractory to prior anti-PD-1 therapy. Ultimately, among the nine evaluable cHL patients, four achieved CR and five had a PR. Perhaps most notably, these data suggest that patients with refractory disease to anti-PD-1 therapy should not necessarily be excluded from receiving further treatment with anti-PD-1 treatment, particularly when administered in combination with other agents.

A 2020 phase Ib dose-escalating study further explored the benefits of combination therapy in 30 patients with relapsed/refractory $\mathrm{CHL}^{28}{ }^{2}$ In this trial, patients received pembrolizumab in combination with AFM13, a bispecific tetravalent antibody that targets CD30 on tumor cells and CD16A on natural killer cells and macrophages to induce tumor cell killing. In this study, patients who had received prior anti-PD-1, anti-PD-L1, or anti-PD-1-L2 treatment were excluded. Overall, the study population had an $83 \%$ objective response rate, with $37 \%$ showing a complete metabolic response and $47 \%$ a partial metabolic response. Among those who received the highest treatment dose, the objective response rate was $88 \%$.

Bolstered by the encouraging data above, two clinical trials investigating anti-PD-1 therapy in combination with radiotherapy for patients with relapsed/refractory $\mathrm{cHL}$ are ongoing. NCT04419441 is exploring whether radiotherapy will improve the rate of complete remission in patients receiving anti-PD-L1 therapy. NCT03480334 has the same hypothesis, but its study population is comprised specifically of patients who have recently progressed on anti-PD-1 therapy.

\section{CPI for Maintenance Strategy}

A multicohort phase II study by Armand et al evaluated pembrolizumab as a maintenance strategy post-ASCT. These patients consisted of a particularly high-risk group, with $90 \%$ of the 30 patients deemed high risk by clinical criteria. The maintenance pembrolizumab therapy lasted for eight cycles every 3 weeks. Among 28 evaluable patients, the rate of PFS was $82 \%$ at 18 months, satisfying the investigators' hypothesis that pembrolizumab would improve PFS from $60 \%$ to $80 \%$. ${ }^{29}$ Similarly, maintenance nivolumab (6 months) post-ASCT was also tested in patients with Hodgkin lymphoma (HL). ${ }^{30}$ The data are immature, but out of the 37 patients enrolled on this trial, the 6-month or end of therapy PFS is $92.1 \%$ and the 12-month OS is $100 \%$. The median PFS and OS have not been reached, with a median follow-up of 9.2 months. Only four discontinued the nivolumab owing to adverse events and two patients progressed while on the nivolumab. Both of these trials suggest that maintenance CPIs are tolerable, and mature data are needed for them to become the standard of care. 


\section{Second-Line Therapy}

Several studies have also demonstrated the effectiveness of checkpoint inhibition in combination with other agents as second-line therapy in cHL. In one phase I/II study, a total of 91 patients with relapsed/refractory disease were treated with combination BV plus nivolumab. Patients in this study were divided into two cohorts: one group that received BV and nivolumab in a staggered schedule, and a second group that received therapy concurrently. Therapy proved effective in both cohorts; the rate of CR for treated patients was $67 \%$ and the objective response rate was $85 \% .{ }^{31,32}$ Given these encouraging results, BV plus nivolumab is now approved for use as second-line therapy in relapsed/refractory cHL, establishing an alternative to chemotherapy for consolidation prior to ASCT.

Another trial addressed the question of whether immunotherapy in combination with chemotherapy could safely be tolerated prior to ASCT, without adversely affecting peripheral blood progenitor cell mobilization and engraftment. Patients were treated with a regimen of pembrolizumab plus ifosfamide, carboplatin, and etoposide (ICE). Among the 40 patients with relapsed/refractory cHL recruited, 23 were evaluable. All but one patient in this group had successful stem cell mobilization and harvest (this patient had a severe allergic response to filgrastim and instead had a bone marrow harvest), and all patients who underwent stem cell reinfusion successfully engrafted, with a median time of 11 days for absolute neutrophil recovery and 12 days for platelet recovery. Notably, the therapy was well tolerated in this group, with no reports of the more serious adverse immune CPI toxicities of pneumonitis, colitis, hepatitis, or endocrinopathies. ${ }^{33}$ Therefore, these data further support the feasibility of immunotherapy as second-line therapy in relapsed/refractory cHL prior to ASCT.

The randomized, open-label phase III trial entitled KEYNOTE-204 demonstrated that pembrolizumab monotherapy was superior to $\mathrm{BV}$ in patients with relapsed/refractory $\mathrm{cHL} .{ }^{34}$ This trial was a head-to-head comparison of pembrolizumab and BV, and included both patients with and those without prior BV exposure. All participants were either postASCT or ineligible for transplant. In a head-to-head comparison, patients who received pembrolizumab had higher rates of PFS compared to those who had received BV (HR 0.65, 95\% CI 0.46-0.85), with PFS rates of $53.9 \%$ in patients given pembrolizumab versus $35.6 \%$ in the BV group. Pembrolizumab was superior in all of the study's subgroups, including those who had not previously undergone ASCT, those with primary refractory disease, prior exposure to BV, and no prior exposure to BV. This study led to the extended FDA approval in 2020 of pembrolizumab for adult cHL patients as second-line therapy and pediatric patients as third-line therapy.

In a similar vein, a subsequent phase II study investigated whether pembrolizumab plus gemcitabine, vinorelbine, and liposomal doxorubicin (GVD) would be effective as second-line therapy prior to ASCT. Thirty-seven patients were recruited for the study, and 34 were eligible for analysis. Among this group, 31 achieved a CR after two cycles and three achieved a PR. One of these three partial responders ultimately achieved CR after four cycles of therapy. At the time of publication, 32 patients have undergone ASCT, one is awaiting transplantation, and one declined transplantation and instead opted for maintenance pembrolizumab therapy. All patients were still in remission at the time of publication. ${ }^{35}$ No dose-limiting toxicities were noted in the safety phase of the trial. Grade 3 toxicities include elevated liver enzymes (four patients), neutropenia (four patients), oral mucositis (two patients), rash (one patient), and hyperthyroidism (one patient).

One study pursued a unique angle by having a cohort of patients who received a combination of immunotherapy agents: nivolumab and ipilimumab. Ipilimumab is a monoclonal antibody targeted against CTLA-4, an immune checkpoint receptor on T cells. ${ }^{36}$ Using multiplex immunofluorescence microscopy with digital image analysis, Patel et al found that the tumor microenvironment in cHL is enriched for non-T-regulatory, cytotoxic T-lymphocyte-associated protein-4 (CTLA-4)-positive T cells that outnumber PD-1-positive T cells. ${ }^{37}$ Unlike the tumor-associated macrophages that express PD-L1, T cells that surround the RSCs are more often positive for CTLA-4 than PD-1. The patients included in this trial had wide variance in prior treatments; some had as few as one prior line of therapy while others had received nine lines of therapy (median of 2). Of the 64 patients enrolled in this phase I trial, 61 were evaluable. These patients were separated into three cohorts: one group received BV and ipilimumab; a second received BV and nivolumab; and the final group received BV, ipilimumab, and nivolumab. Each of these cohorts demonstrated robust objective response rates and $\mathrm{CR}$ rates: $76 \%$ and $57 \%$, respectively, in the BV/ipilimumab group; $88 \%$ and $61 \%$, respectively, in the BV/ nivolumab group; and $82 \%$ and $73 \%$, respectively, in the BV/nivolumab/ipilimumab group. 
The most significant toxicities occurred in the BV/nivolumab and BV/nivolumab/ipilimumab groups, each of which had one grade 5 pneumonitis death and five dose-limiting toxicities between the two of them. Common grade 1-2 toxicities included fatigue, elevation of liver transaminases, peripheral neuropathy, and diarrhea. For grade 3 or 4 adverse events, $10(43 \%)$ of the ipilimumab group, three (16\%) of the nivolumab group, and $11(50 \%)$ of the triple-therapy patients experienced high-grade toxicities.

\section{Frontline Therapy}

More recently, Allen et al pursued CPI therapy as a component of frontline therapy, given its effectiveness in relapsed/ refractory disease. ${ }^{38}$ In this multicenter phase II study, 30 patients with untreated, early, unfavorable, or advanced-stage disease were treated with pembrolizumab and doxorubicin, vinblastine, and dacarbazine (AVD) chemotherapy. These patients first received three cycles of pembrolizumab monotherapy, followed by AVD for four to six cycles depending on the stage and bulk of disease. After pembrolizumab monotherapy, 11 patients had a complete metabolic response, and an additional seven patients had $>90 \%$ reduction in metabolic tumor volume based on PET/CT. All patients in the study attained a complete metabolic response after two cycles of AVD, and at median follow-up of 22.5 months there were no cases of disease progression, death, or further therapy. Furthermore, the therapy was well tolerated, with the most notable adverse effects being six patients with grade 1 rash, four patients with grade 2 transfusion reactions, one patient with reversible grade 4 transaminitis, and one patient with reversible Bell's palsy. ${ }^{39}$

This trial by Allen et al used a lead-in or sequential strategy of CPIs prior to chemotherapy, with high response rates. However, the question of concurrent versus sequential use of CPIs with chemotherapy remains. The German Hodgkin Study Group led by Bröckelmann et al performed a phase II study looking at early-stage unfavorable disease patients, with a 1:1 assignment to either concurrent treatment with four cycles of nivolumab and AVD or sequential treatment with four doses of nivolumab, two cycles of N-AVD, and two cycles of AVD, followed by involved-site radiotherapy. ${ }^{40}$ There were 109 patients included in this study, with a 90\% CR rate in the concurrent arm and 94\% CR in the sequential arm. The one-year PFS rates were $100 \%$ and $98 \%$ for the concurrent arm and sequential arm, respectively. These results suggest that both options are reasonable, but the single-agent nivolumab (four cycles) lead-in had a $96 \%$ response rate, which, along with the pembrolizumab data from Allen et $\mathrm{al},{ }^{39}$ will require further investigation to minimize toxicities.

The CheckMate 205 trial referenced above (in the section "Third or Greater Line of Therapy") also included a cohort of patients who received immunotherapy with chemotherapy as frontline therapy for newly diagnosed cHL. ${ }^{41,42}$ In this study, 51 patients with stage IIB with unfavorable risk, III, or IV cHL underwent treatment. The investigators planned for four doses of nivolumab monotherapy followed by nivolumab plus doxorubicin, vinblastine, and dacarbazine combination therapy for six cycles. After two combination cycles, the rate of complete remission was $51 \%$ (and $71 \%$ complete metabolic response) via an independent review committee and $71 \%$ per investigator. On a more recent trial result update, the ORR was $84 \%$ (95\% CI 71-93\%), including $67 \%$ of the patients reaching a CR. ${ }^{43}$ At 21 -month follow-up, the rate of PFS was $83 \%$ per investigator. Furthermore, Roemer et al found that responses in this trial correlated with a higher level of 9p24.1 copy gain and increased PD-L1 expression on RSCs. ${ }^{44}$ Surprisingly, major histocompatibility complex (MHC) class II on the RSCs was predictive of the responses, whereas $\beta_{2}$-microglobulin/MHC class I was not.

\section{Other Checkpoint Inhibitors}

Though other anti-PD-1 immunotherapy agents have not been approved by the FDA for use in relapsed/refractory cHL, studies indicate that sintilimab, tislelizumab, and camrelizumab are effective in these patients. Sintilimab, a fully humanized $\mathrm{IgG}_{4}$ monoclonal antibody toward PD-1, was administered to patients taking part in ORIENT-1, a phase II trial consisting of 96 patients from 18 hospitals in China who had relapsed/refractory disease following two or more lines of therapy. ${ }^{45}$ In total, 74 patients among the 92 analyzed (80.4\%, 95\% CI 70.9-88.0\%) demonstrated an objective response via review from an independent committee. In another phase II study among 70 Chinese patients with relapsed/ refractory HL who received tislelizumab, a humanized anti-PD-1 antibody that is designed to bind less to Fc gamma receptors, $61(87.1 \%)$ of the patients assessed by an independent review committee had an objective response, with a 9month PFS rate of $74.5 \%{ }^{46}$ 


\begin{tabular}{|c|c|c|c|c|c|c|c|c|c|c|c|c|c|}
\hline$\hat{b}$ & 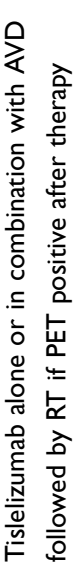 & 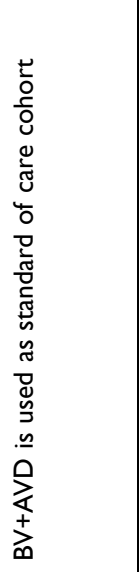 & 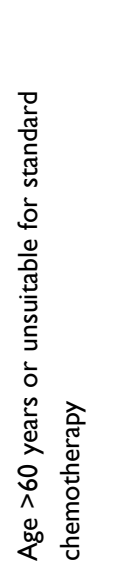 & 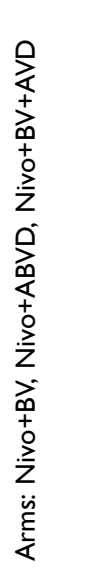 & 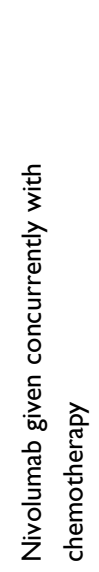 & 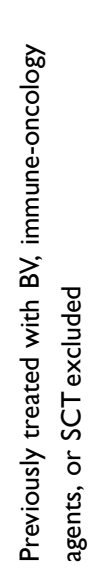 & 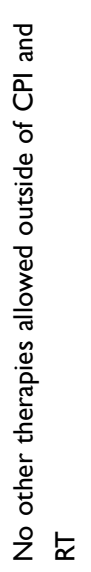 & 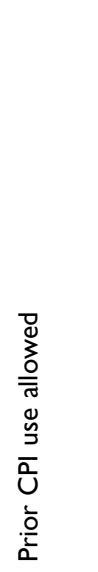 & 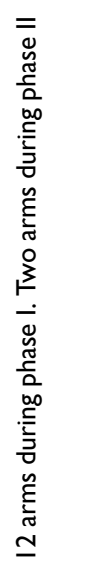 & 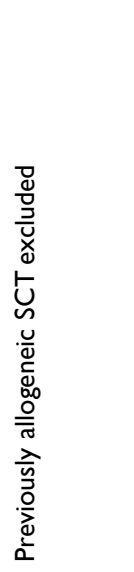 & 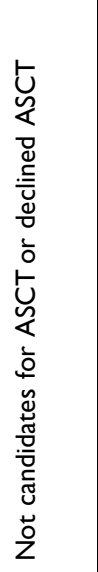 & 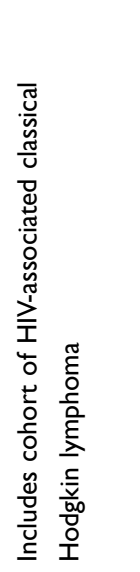 & 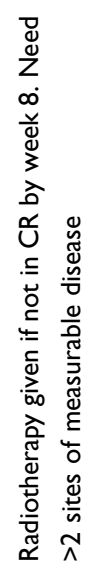 \\
\hline 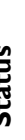 & 承 & 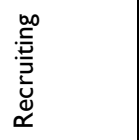 & 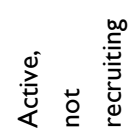 & 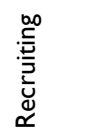 & 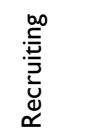 & 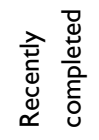 & 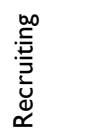 & 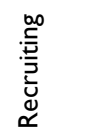 & 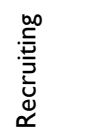 & 总 & 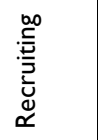 & 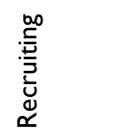 & 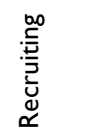 \\
\hline j & 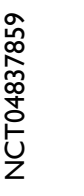 & 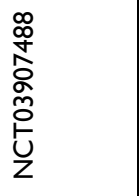 & 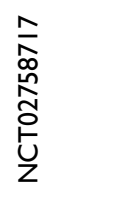 & 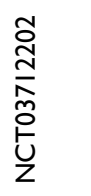 & 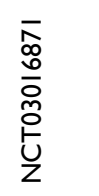 & $\begin{array}{l}\frac{\hat{o}}{N} \\
\hat{N} \\
\tilde{\delta} \\
z \\
z\end{array}$ & 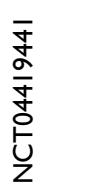 & 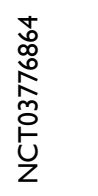 & $\begin{array}{l}\sigma \\
\alpha \\
o \\
o \\
\frac{0}{0} \\
\frac{0}{z}\end{array}$ & 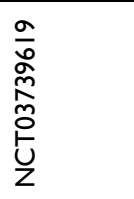 & 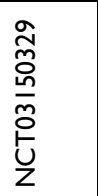 & 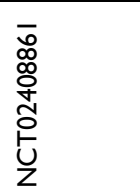 & 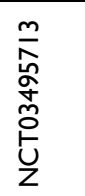 \\
\hline v & $=$ & $\equiv$ & $=$ & $=$ & $=$ & $\equiv$ & $\propto$ & $=$ & $\equiv$ & $\equiv$ & - & - & $=$ \\
\hline שֶّ & 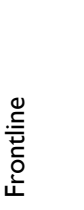 & 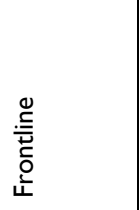 & 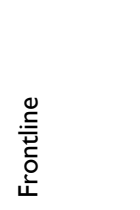 & 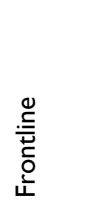 & 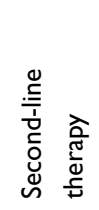 & 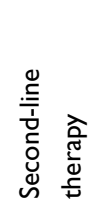 & 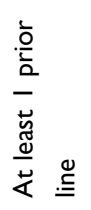 & 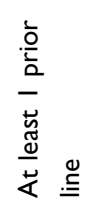 & 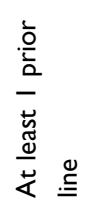 & 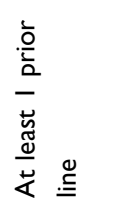 & 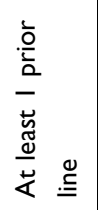 & 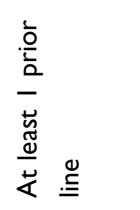 & 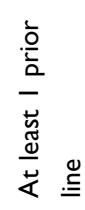 \\
\hline & 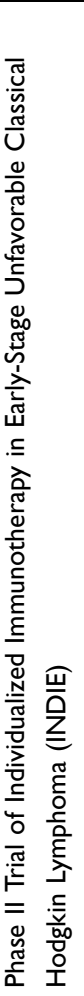 & 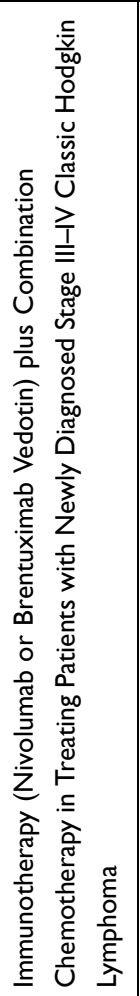 & 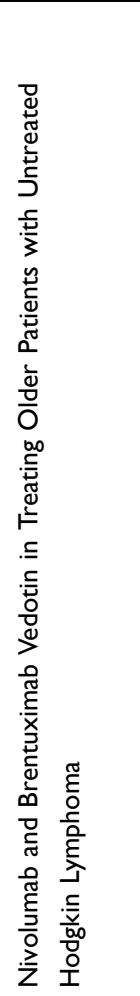 & 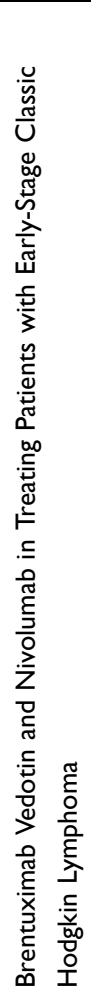 & 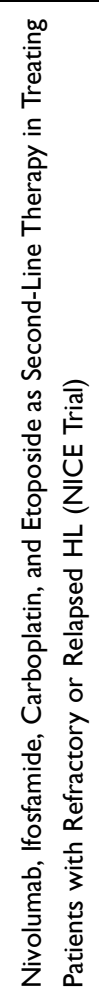 & 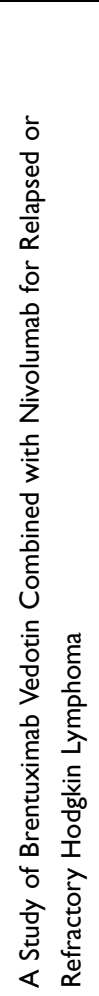 & 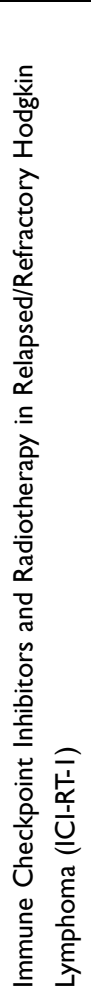 & 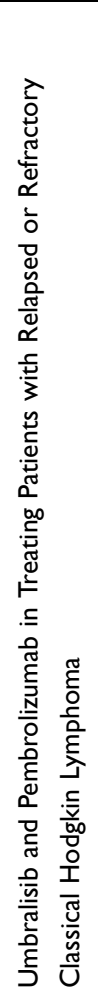 & 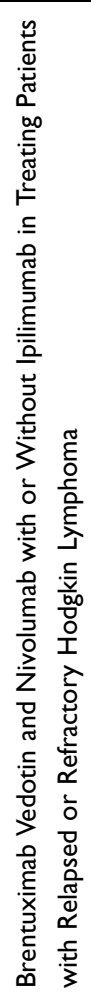 & 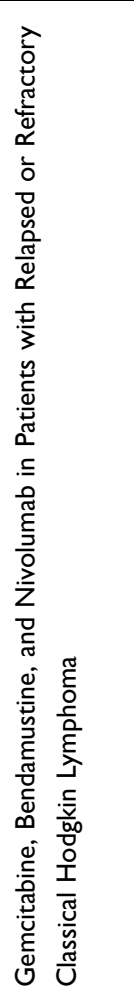 & 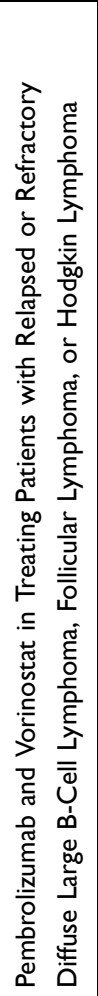 & 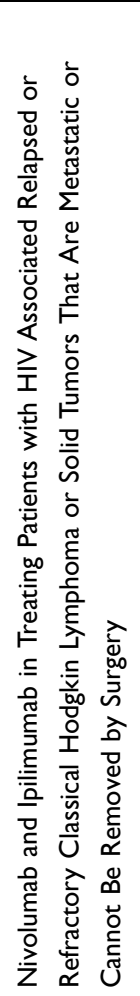 & 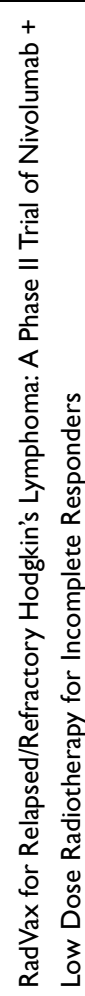 \\
\hline
\end{tabular}




\begin{tabular}{|c|c|c|c|c|c|}
\hline 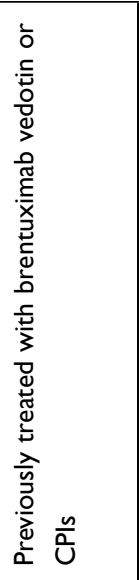 & 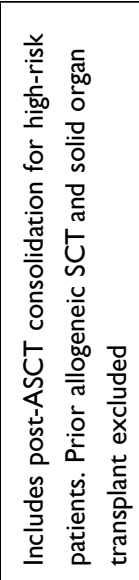 & 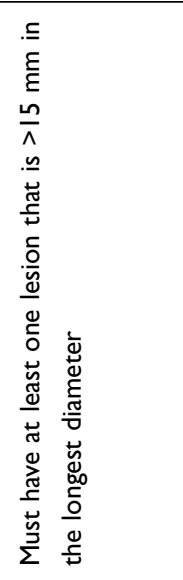 & 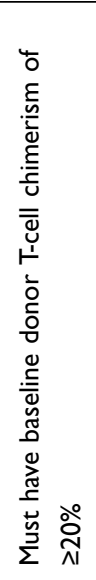 & 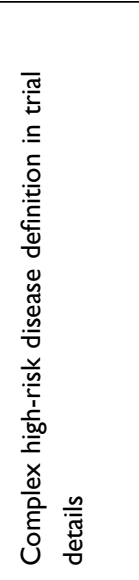 & 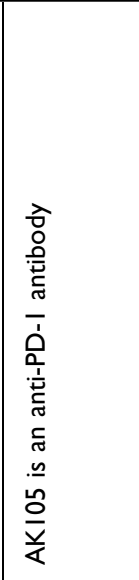 \\
\hline 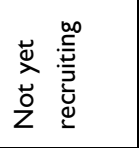 & 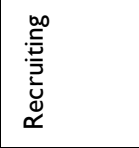 & 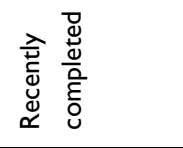 & 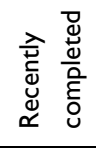 & 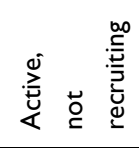 & $\begin{array}{l}\frac{5}{3} \\
\frac{0}{5} \\
\frac{5}{5}\end{array}$ \\
\hline 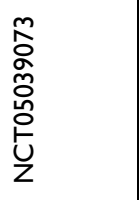 & 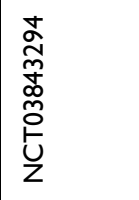 & 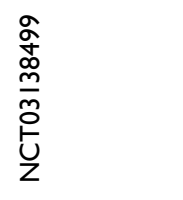 & 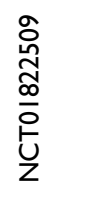 & 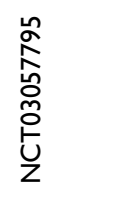 & 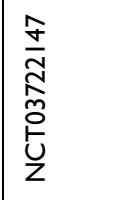 \\
\hline$=$ & - & $\equiv$ & - & $=$ & $\equiv$ \\
\hline 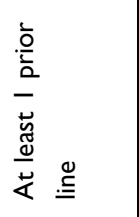 & 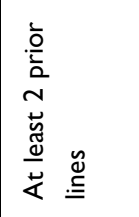 & 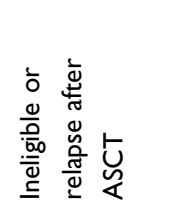 & 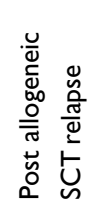 & 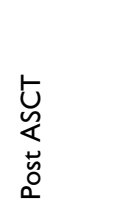 & 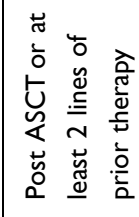 \\
\hline 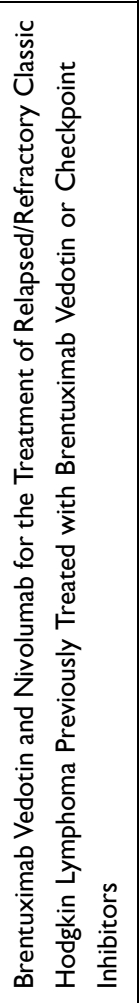 & 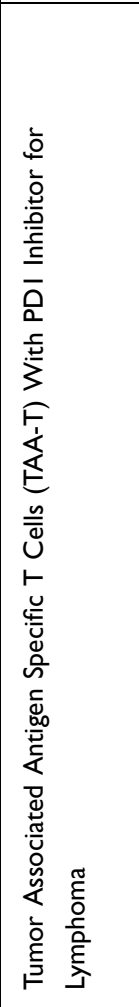 & 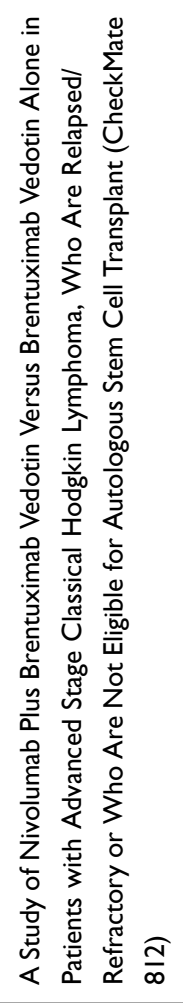 & 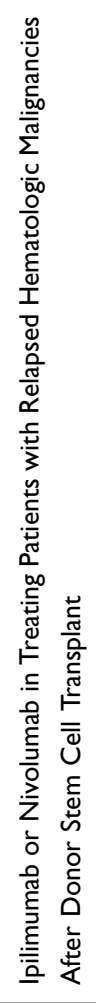 & 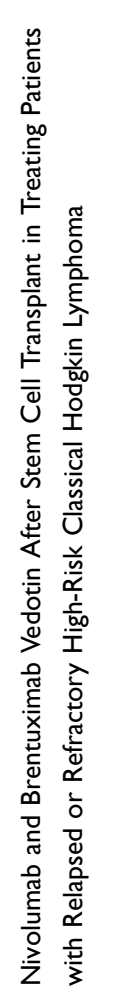 & 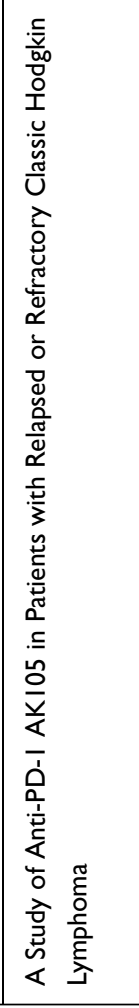 \\
\hline
\end{tabular}


Camrelizumab, another anti-PD-1 antibody, was administered to 75 Chinese patients with relapsed/refractory cHL after ASCT or who had received two or more lines of chemotherapy, among whom 57 (76.0\%, 95\% CI 64.7-85.1\%) achieved an objective response based on review by an independent review committee. ${ }^{47} \mathrm{~A}$ two-arm, open-label phase II study consisting of $86 \mathrm{relapsed} /$ refractory cHL patients who had already undergone at least two lines of therapy reaffirmed the efficacy of camrelizumab, though it also suggested that co-administration of decitabine improves the clinical response. Among the patients who were naïve to anti-PD-1 therapy, six of 19 (32\%) achieved complete remission with camrelizumab monotherapy, compared with 30 of 42 patients (71\%) who received decitabine plus camrelizumab. In patients who had previously been treated with anti-PD-1 therapy, 28\% achieved complete remission, while PR was seen in $24 \%$ of these patients, suggesting that co-administration of decitabine could bypass PD-1 resistance in patients who had previously been treated with anti-PD-1 therapy. ${ }^{48}$

JAVELIN Hodgkins, a phase Ib trial for patients with relapsed/refractory cHL, showed that avelumab, an anti-PD-L1 monoclonal antibody, is also effective in this patient population. ${ }^{49}$ In this study of 31 heavily pretreated patients, nine had received three prior treatments and 20 had received four or more treatments. The objective response rate among all patients was $41.9 \%$, with a CR rate of $19.4 \%$, and the objective response rate among patients who had previously received an allogeneic hematopoietic stem cell transplant was even more significant, at 55.6\%.

\section{Conclusion}

CPIs are destined to be active agents in HL owing to the biological nature of 9p24.1 amplification. Both pembrolizumab and nivolumab have been successful in treating $\mathrm{cHL}$ patients as single agents or as combination therapy with brentuximab vedotin or chemotherapy in the third line. Growing evidence suggests that CPIs in the first or second line may improve outcomes. Additional clinical trials are needed and are ongoing to evaluate the best time to use CPIs in these patient populations (Table 1). CPIs in combination with newer targeting agents will also be a point of further trials, especially since the PD-1/PD-2 pathway is not the only method of immune evasion by RSCs. ${ }^{50}$ Furthermore, a better understanding of the systemic and tumor microenvironment will help us predict which patients will respond to CPI therapy. ${ }^{51}$

\section{Disclosure}

Dr. Leo I Gordon reports consulting fees from Astra Zeneca, consulting fees from Karyopharm Therapeutics, consulting fees from Epizyme, consulting fees from Janssen Research and Development, and consulting fees from Janssen Research and Development, outside the submitted work; in addition, Dr Leo I Gordon has a patent for Gold Nanoparticles for Lymphoma, issued to Application Serial No. 62/902,342. The authors report no other conflicts of interest in this work.

\section{References}

1. Hodgkin Lymphoma Cancer stat facts; [cited 2022 February 10]. Available from: https://seer.cancer.gov/statfacts/html/ hodg.html

2. Hodgkin Lymphoma. NCCN clinical practice guidelines in oncology (NCCN guidelines ${ }^{\circledR}$ ) NCCN.org NCCN guidelines for patients ${ }^{\circledR}$; 2021. Available from: www.nccn.org/patients. Accessed February 2, 2022.

3. van Leeuwen FE, Ng AK. Late sequelae in Hodgkin lymphoma survivors. Hematol Oncol. 2017;35:60-66. doi:10.1002/hon.2402

4. van Nimwegen FA, Ntentas G, Darby SC. Risk of heart failure in survivors of Hodgkin lymphoma: effects of cardiac exposure to radiation and anthracyclines. Blood. 2017;129(16):2257-2265. doi:10.1182/blood-2016-09-740332

5. Connors JM, Jurczak W, Straus DJ, et al. Brentuximab vedotin with chemotherapy for stage III or IV Hodgkin's lymphoma. $N$ Engl $J$ Med. 2018;378(4):331-344. doi:10.1056/NEJMoa1708984

6. Straus DJ, Długosz-Danecka M, Connors JM, et al. Brentuximab vedotin with chemotherapy for stage III or IV classical Hodgkin lymphoma (ECHELON-1): 5-year update of an international, open-label, randomised, phase 3 trial. Lancet Haematol. 2021;8(6):e410-e421. doi:10.1016/ S2352-3026(21)00102-2

7. Zelenetz AD, Hamlin P, Kewalramani T, et al. Ifosfamide, carboplatin, etoposide (ICE)-based second-line chemotherapy for the management of relapsed and refractory aggressive non-Hodgkin's lymphoma. Ann Oncol. 2003;14(SUPPL. 1):i5-i10. doi:10.1093/annonc/mdg702

8. Bartlett NL, Niedzwiecki D, Johnson JL, et al. Gemcitabine, vinorelbine, and pegylated liposomal doxorubicin (GVD), a salvage regimen in relapsed Hodgkin's lymphoma: CALGB 59804. Ann Oncol. 2007;18(6):1071-1079. doi:10.1093/annonc/mdm090

9. Castagna L, Santoro A, Carlo-Stella C. Salvage therapy for Hodgkin's lymphoma: a review of current regimens and outcomes. J Blood Med. 2020;11:389-403. doi:10.2147/JBM.S250581

10. Chen BJ, Chapuy B, Ouyang J, et al. PD-L1 expression is characteristic of a subset of aggressive B-cell lymphomas and virus-associated malignancies. Clin Cancer Res. 2013;19(13):3462-3473. doi:10.1158/1078-0432.CCR-13-0855 
11. Cader FZ, Schackmann RCJ, Hu X, et al. Mass cytometry of Hodgkin lymphoma reveals a CD4+ regulatory T-cell-rich and exhausted T-effector microenvironment. Blood. 2018;132(8):825-836. doi:10.1182/blood-2018-04-843714

12. Carey CD, Gusenleitner D, Lipschitz M, et al. Topological analysis reveals a PD-L1-associated microenvironmental niche for Reed-Sternberg cells in Hodgkin lymphoma. Blood. 2017;130(22):2420-2430. doi:10.1182/blood-2017-03-770719

13. Roemer MG, Advani RH, Ligon AH, et al. PD-L1 and PD-L2 genetic alterations define classical Hodgkin lymphoma and predict outcome. $J$ Clin Oncol. 2016;34(23):2690-2697. doi:10.1200/JCO.2016.66.4482

14. Wienand K, Chapuy B, Stewart C, et al. Genomic analyses of flow-sorted Hodgkin Reed-Sternberg cells reveal complementary mechanisms of immune evasion. Blood Adv. 2019;3(23):4065-4080. doi:10.1182/bloodadvances.2019001012

15. Batlevi CL, Matsuki E, Brentjens RJ, Younes A. Novel immunotherapies in lymphoid malignancies. Nat Rev Clin Oncol. 2015;13(1):25-40. doi:10.1038/nrclinonc. 2015.187

16. Pezeshki PS, Eskian M, Hamblin MR, Rezaei N. Immune checkpoint inhibition in classical Hodgkin lymphoma. Expert Rev Anticancer Ther. 2021;00(00):1-14.

17. Al-Hadidi SA, Chuang HH, Miranda RN, Lee HJ. Programmed cell death-one inhibition therapy in classical Hodgkin lymphoma. Clin Lymphoma Myeloma Leuk. 2021;21(2):e105-e111. doi:10.1016/j.clml.2020.08.031

18. Savage KJ, Steidl C. Immune checkpoint inhibitors in Hodgkin and non-Hodgkin lymphoma: how they work and when to use them. Expert Rev Hematol. 2016;9(11):1007-1009. doi:10.1080/17474086.2016.1242404

19. Lin RJ, Diefenbach CS. Checkpoint inhibition in Hodgkin lymphoma: saving the best for last? Oncology (Williston Park). 2016;30(10):914-920.

20. Ansell SM, Lesokhin AM, Borrello I, et al. PD-1 blockade with nivolumab in relapsed or refractory Hodgkin's lymphoma. $N$ Engl J Med. 2015;372 (4):311-319. doi:10.1056/NEJMoa1411087

21. Maruyama D, Hatake K, Kinoshita T, et al. Multicenter phase II study of nivolumab in Japanese patients with relapsed or refractory classical Hodgkin lymphoma. Cancer Sci. 2017;108(5):1007-1012. doi:10.1111/cas.13230

22. Younes A, Santoro A, Shipp M, et al. Nivolumab for classical Hodgkin's lymphoma after failure of both autologous stem-cell transplantation and brentuximab vedotin: a multicentre, multicohort, single-arm phase 2 trial. Lancet Oncol. 2016;17(9):1283-1294. doi:10.1016/S1470-2045(16) 30167-X

23. Armand P, Shipp MA, Ribrag V, et al. Programmed death-1 blockade with pembrolizumab in patients with classical Hodgkin lymphoma after brentuximab vedotin failure. J Clin Oncol. 2016;34(31):3733-3739. doi:10.1200/JCO.2016.67.3467

24. Chen R, Zinzani PL, Fanale MA, et al. Phase II study of the efficacy and safety of pembrolizumab for relapsed/refractory classic Hodgkin lymphoma. J Clin Oncol. 2017;35(19):2125-2132. doi:10.1200/JCO.2016.72.1316

25. Armand P, Zinzani PLL, Lee HJ, et al. Five-year follow-up of keynote-087: pembrolizumab monotherapy in relapsed/refractory classical Hodgkin lymphoma (R/R cHL). Blood. 2021;138(Supplement 1):1366. doi:10.1182/blood-2021-147881

26. Armand P, Kuruvilla J, Michot JM, et al. KEYNOTE-013 4-year follow-up of pembrolizumab in classical Hodgkin lymphoma after brentuximab vedotin failure. Blood Adv. 2020;4(12):2617-2622. doi:10.1182/bloodadvances.2019001367

27. Herrera AF, Chen L, Popplewell LL, et al. Preliminary results from a phase I trial of pembrolizumab plus vorinostat in patients with relapsed or refractory diffuse large B-cell lymphoma, follicular lymphoma, and Hodgkin lymphoma. Blood. 2019;134(Supplement_1):759. doi:10.1182/blood2019-123163

28. Bartlett NL, Herrera AF, Domingo-Domenech E, et al. A phase 1b study of AFM13 in combination with pembrolizumab in patients with relapsed or refractory Hodgkin lymphoma. Blood. 2020;136(21):2401-2409. doi:10.1182/blood.2019004701

29. Armand P, Bin CY, Redd RA, et al. PD-1 blockade with pembrolizumab for classical Hodgkin lymphoma after autologous stem cell transplantation. Blood. 2019;134(1):22-29. doi:10.1182/blood.2019000215

30. Bachier C, Schade H, Zoghi B, Ramakrishnan A, Shah NN. A phase II single arm study of nivolumab as maintenance therapy after autologous stem cell transplantation in patients with Hodgkin lymphoma at risk of relapse or progression. Blood. 2021;138(Supplement 1):2455. doi:10.1182/blood2021-148139

31. Herrera AF, Moskowitz AJ, Bartlett NL, et al. Interim results of brentuximab vedotin in combination with nivolumab in patients with relapsed or refractory Hodgkin lymphoma. Blood. 2018;131(11):1183-1194. doi:10.1182/blood-2017-10-811224

32. Moskowitz AJ, Advani RH, Bartlett NL, et al. Brentuximab vedotin and nivolumab for relapsed or refractory classic Hodgkin lymphoma: long-term follow-up results from the single-arm phase 1/2 study. Blood. 2019;134(Supplement_1):238. doi:10.1182/blood-2019-122576

33. Bryan LJ, Smith SE, Allen P, et al. Safety and toxicity profile of pembrolizumab (PEM) in combination with ICE chemotherapy followed by autologous stem cell transplantation for relapsed/refractory classical Hodgkin lymphoma: no impairment in stem cell mobilization or engraftment. Blood. 2019;134(Supplement_1):4029. doi:10.1182/blood-2019-123879

34. Kuruvilla J, Ramchandren R, Santoro A, et al. KEYNOTE-204: randomized, open-label, phase III study of pembrolizumab (pembro) versus brentuximab vedotin (BV) in relapsed or refractory classic Hodgkin lymphoma (R/R cHL). ASCO Ann Meet. 2020;38(15_suppl):8005. doi:10.1200/ JCO.2020.38.15_suppl.8005

35. Moskowitz AJ, Shah G, Schöder H, et al. Phase II trial of pembrolizumab plus gemcitabine, vinorelbine, and liposomal doxorubicin as second-line therapy for relapsed or refractory classical Hodgkin lymphoma. J Clin Oncol. 2021;39:3109-3117. doi:10.1200/JCO.21.01056

36. Diefenbach CS, Hong F, Ambinder RF, et al. Ipilimumab, nivolumab, and brentuximab vedotin combination therapies in patients with relapsed or refractory Hodgkin lymphoma: phase 1 results of an open-label, multicentre, phase 1/2 trial. Lancet Haematol. 2020;7(9):e660-e670. doi:10.1016/ S2352-3026(20)30221-0

37. Patel SS, Weirather JL, Lipschitz M, et al. The microenvironmental niche in classic Hodgkin lymphoma is enriched for CTLA-4-positive T cells that are PD-1-negative. Blood. 2019;134(23):2059-2069. doi:10.1182/blood.2019002206

38. Alencar AJ, Moskowitz CH. Immune-checkpoint inhibition as first-line therapy for Hodgkin lymphoma. Nat Rev Clin Oncol. 2019;16(10):599-600. doi:10.1038/s41571-019-0255-8

39. Allen PB, Savas H, Evens AM, et al. Pembrolizumab followed by AVD in untreated early unfavorable and advanced-stage classical Hodgkin lymphoma. Blood. 2021;137(10):1318-1326. doi:10.1182/blood.2020007400

40. Bröckelmann PJ, Goergen H, Keller U. Efficacy of nivolumab and AVD in early-stage unfavorable classic Hodgkin lymphoma: the randomized phase 2 German Hodgkin study group NIVAHL trial. JAMA Oncol. 2020;6(6):872-880. doi:10.1001/jamaoncol.2020.0750 
41. Ramchandren R, Fanale MA, Rueda A, et al. Nivolumab for newly diagnosed advanced-stage classical Hodgkin lymphoma (cHL): results from the phase 2 checkmate 205 study. Blood. 2017;130(Suppl 1):651. doi:10.1182/blood.V130.Suppl_1.651.651

42. Ansell S, Ramchandren R, Domingo-Domènech E, et al. Nivolumab plus doxorubicin, vinblastine and dacarbazine for newly diagnosed advancedstage classical Hodgkin lymphoma: checkmate 205 cohort D 2-year follow-up. Hematol Oncol. 2019;37:146-147. doi:10.1002/hon.104_2629

43. Ramchandren R, Domingo-Domènech E, Rueda A. Nivolumab for newly diagnosed advanced-stage classic Hodgkin lymphoma: safety and efficacy in the phase II checkMate 205 study. J Clin Oncol. 2019;37(23):1997-2007. doi:10.1200/JCO.19.00315

44. Roemer MGM, Redd RA, Cader FZ, et al. Major histocompatibility complex class II and programmed death ligand 1 expression predict outcome after programmed death 1 blockade in classic Hodgkin lymphoma. J Clin Oncol. 2018;36(10):942-950. doi:10.1200/JCO.2017.77.3994

45. Shi Y, Su H, Song Y, et al. Safety and activity of sintilimab in patients with relapsed or refractory classical Hodgkin lymphoma (ORIENT-1): a multicentre, single-arm, phase 2 trial. Lancet Haematol. 2019;6(1):e12-e19. doi:10.1016/S2352-3026(18)30192-3

46. Song Y, Gao Q, Zhang H. Treatment of relapsed or refractory classical Hodgkin lymphoma with the anti-PD-1, tislelizumab: results of a phase 2, single-arm, multicenter study. Leukemia. 2020;34(2):533-542. doi:10.1038/s41375-019-0545-2

47. Song Y, Wu J, Chen X. A single-arm, multicenter, phase II study of camrelizumab in relapsed or refractory classical Hodgkin lymphoma. Clin Cancer Res. 2019;25(24):7363-7369. doi:10.1158/1078-0432.CCR-19-1680

48. Nie J, Wang C, Liu Y, et al. Addition of low-dose decitabine to anti-PD-1 antibody camrelizumab in relapsed/refractory classical Hodgkin lymphoma. J Clin Oncol. 2019;37(17):1479-1489. doi:10.1200/JCO.18.02151

49. Herrera AF, Burton C, Radford J, et al. Avelumab in relapsed/refractory classical Hodgkin lymphoma: phase 1b results from the JAVELIN Hodgkins trial. Blood Adv. 2021;5(17):3387-3396. doi:10.1182/bloodadvances.2021004511

50. Green MR, Monti S, Rodig SJ, et al. Integrative analysis reveals selective 9p24.1 amplification, increased PD-1 ligand expression, and further induction via JAK2 in nodular sclerosing Hodgkin lymphoma and primary mediastinal large B-cell lymphoma. Blood. 2010;116(17):3268-3277. doi:10.1182/blood-2010-05-282780

51. Cader FZ, Hu X, Goh WL, et al. A peripheral immune signature of responsiveness to PD-1 blockade in patients with classical Hodgkin lymphoma. Nat Med. 2020;26(9):1468-1479. doi:10.1038/s41591-020-1006-1

\section{Publish your work in this journal}

ImmunoTargets and Therapy is an international, peer-reviewed open access journal focusing on the immunological basis of diseases, potential targets for immune based therapy and treatment protocols employed to improve patient management. Basic immunology and physiology of the immune system in health, and disease will be also covered. In addition, the journal will focus on the impact of management programs and new therapeutic agents and protocols on patient perspectives such as quality of life, adherence and satisfaction. The manuscript management system is completely online and includes a very quick and fair peer-review system, which is all easy to use. Visit http://www.dovepress.com/testimonials.php to read real quotes from published authors.

Submit your manuscript here: http://www.dovepress.com/immunotargets-and-therapy-journal 